\title{
Involvement of Membrane GRP78 in Trophoblastic Cell Fusion
}

\author{
Sarah Fradet ${ }^{1}$, Sandra Pierredon ${ }^{1}$, Pascale Ribaux ${ }^{1}$, Manuella Epiney ${ }^{1}$, Kazuo Shin Ya ${ }^{2}$, Olivier Irion ${ }^{1}$, \\ Marie Cohen ${ }^{1 *}$
}

1 Department of Gynecology Obstetrics, Faculty of Medicine, Geneva, Switzerland, 2 Biomedicinal Information Research Center, National Institute of Advanced Industrial Science and Technology, Tokyo, Japan

\begin{abstract}
Background: Glucose-regulated protein 78 (GRP78) is highly expressed in first trimester cytrophoblastic cells (CTBs), especially in syncytiotrophoblast (STB). However, the role of GRP78 in these cells has never been investigated.

Methodology/Principal Findings: In this study, we have examined the role of GRP78 in trophoblast fusion using the Bewo choriocarcinoma cell line as a model of cytotrophoblast fusion. Down regulation of GRP78 by siRNA or chemical inhibitors and use of antibodies against GRP78 in culture medium significantly decreased forskolin-induced fusion capacity of Bewo cells suggesting the involvement of membrane GRP78 in trophoblast fusion. GRP78 expression was also studied in preeclamptic (PE) CTBs which are known to have lower fusion capacity compared to control CTBs. Interestingly, despite the increase of GRP78 mRNA in PE CTBs, membrane GRP78 is significantly decreased in PE CTBs compared to control CTBs, suggesting that relocation of GRP78 from the endoplasmic reticulum to cell surface is probably altered in PE CTBs.

Conclusions: Our results imply that membrane GRP78 could play an important role in syncytialisation. They also suggest that deregulation of GRP78 expression or relocation at cell surface might be involved in pregnancy complication associated with defective syncytialisation, such as preeclampsia.
\end{abstract}

Citation: Fradet S, Pierredon S, Ribaux P, Epiney M, Shin Ya K, et al. (2012) Involvement of Membrane GRP78 in Trophoblastic Cell Fusion. PLoS ONE 7(8): e40596. doi:10.1371/journal.pone.0040596

Editor: Cees Oudejans, VU University Medical Center, The Netherlands

Received March 30, 2012; Accepted June 11, 2012; Published August 9, 2012

Copyright: (C) 2012 Fradet et al. This is an open-access article distributed under the terms of the Creative Commons Attribution License, which permits unrestricted use, distribution, and reproduction in any medium, provided the original author and source are credited.

Funding: This work was supported by the Swiss National Funds (31003A-127392; www.snf.ch) and HUG (PRD 10-I-11). The funders had no role in study design, data collection, and analysis, decision to publish, or preparation of the manuscript.

Competing Interests: The authors have declared that no competing interests exist.

*E-mail: marie.cohen@hcuge.ch

\section{Introduction}

Placenta is a transient, autonomous and multifunctional organ whose main role is to permit feto-maternal exchanges of gas and nutrients [1]. At the feto-maternal interface, trophoblast cells differentiate according to the villous or the extravillous pathway [2]. In the extravillous pathway, extravillous cytotrophoblastic cells (evCTBs) proliferate and differentiate into an invasive phenotype [2]. These cells invade decidual stromal compartments as well as spiral arteries of the decidua and the proximal third of the myometrium [3]. In the villous pathway, villous cytotrophoblastic cells (CTBs) remain in the foetal compartment and fuse to form the syncytiotrophoblast (STB) [4]. STB is a multinuclear tissue forming the outer surface of the foetal part of the placenta and is crucial throughout pregnancy [5]. Indeed, this layer exerts unique specialized functions such as hormone secretion and generation of an immunological barrier [6]. The mechanism involved in vCTB differentiation and fusion into the STB is still unclear. Purified mononucleated $\mathrm{VCTB}$ aggregate and fuse in vitro to form multinucleated STB. This process is induced by treatment with cAMP, or with agents which increase intracellular cAMP levels [7]. The in vitro syncytialisation of human primary vCTB to a STB phenotype has been arbitrarily divided into two stages: the morphological and the biochemical differentiation [8]. The initial stage is referred as morphological differentiation and is accompanied to the aggregation and fusion of vCTB to form syncytium. The second stage is referred as biochemical differentiation and is characterized by expression of genes involved in substrate transport, hormone secretion and other functions of fully differentiated STB.

Glucose-regulated protein of $78 \mathrm{kDa}$ (GRP78) is an endoplasmic reticulum (ER) molecular chaperone that belongs to the heat shock protein 70 (HSP70) family (for a review, see [9]). The primary functions of GRP78 are related to its capacity to bind hydrophobic regions on nascent polypeptides in the ER and to its pivotal role in the signalling cascade producing the unfolded protein response (UPR) [10]. GRP78 expression can be stimulated by a variety of environmental and physiological stress conditions such as glucose starvation or hypoxia $[11,12]$. GRP78 is wellknown to reside inside the ER lumen. However, numerous recent studies show that this chaperone is also located at the membrane of cancer cells and cells undergoing ER stress [13] [12]. The mechanisms responsible for the translocation of this protein from the ER to the plasma membrane remain poorly understood [14]. GRP78 on the outer plasma membrane functions as a receptor for a wide variety of ligands [10] and several small proteins can bind to surface GRP78 and modulate proliferation [13]. Recently, we have demonstrated that trophoblastic GRP78 was mainly found 
on the cell surface where it colocalized with p53 [15]. This distribution pattern of GRP78 and p53 is surprising but reveals another common trait between CTBs and cancer cells [15].

GRP78 protein and autoantibodies were also found in plasma of pregnant women. Interestingly, these autoantibodies were significantly lower in plasma of first trimester pregnant women who will subsequently develop preeclampsia (PE) [16]. Since hypoxia and glucose starvation occur in the first trimester PE placenta, it would be expected that GRP78 is overexpressed in these cells. PE is a two-stage disease characterised by abnormal placentation and vascular remodeling and the subsequent maternal syndrome marked by endothelial injury and activation. This pathology is associated with defects in the invasive pathway [6] and in the STB formation [17]. Immunohistochemistry of first trimester trophoblast also revealed that GRP78 was highly expressed in STB [15]. Since PE is associated with defects in STB formation $[17,18]$ and knowing that GRP78 is present at the membrane of CTBs and is strongly expressed in STB, we have decided to further investigate the possible role of GRP78 at the cell surface in the CTB-STB fusion process called syncytialisation. Because of the crucial role of STB throughout pregnancy, an excess or lack of STB formation could have dramatic consequences for both the mother and the foetus. Thus, it is important to understand the mechanisms leading to its formation. For this purpose, we investigated the role of GRP78 in the cell-fusion-inducible BeWo cell line.

\section{Results}

\section{Expression of GRP78 is upregulated by forskolin treatment in BeWo cells and primary CTBs}

Trophoblastic cells fuse in response to treatment with cAMP [7] [19]. Forskolin is often used to induce cAMP level and fusion of trophoblastic cells [19]. Therefore, we have first examined the effects of forskolin treatment on GRP78 expression in trophoblastic cells. As shown in figure 1, treatment of trophoblastic cells (primary first trimester and term CTBs as well as choriocarcinoma BeWo cell line) with forskolin led to a significant upregulation of GRP78 mRNA (A) and protein (B) expression. Moreover, forskolin-induced GRP78 expression led to increased membrane GRP78 expression in BeWo cells (figure 1C).

\section{Down-regulating GRP78 or blocking membrane GRP78 inhibits cell fusion}

The choriocarcinoma BeWo cell line is the most extensively used cellular in vitro model to study villous trophoblast fusion [20]. This study took advantage of this cell line to investigate the role of membrane GRP78 in trophoblast cell fusion.

We have first validated the efficiency of GRP78 downregulation by a specific siRNA and two chemical inhibitors : versipelostatin (VST) [21] and CB106 (pimprinine). As shown in figure 2, the reduction of GRP78 protein expression was on average 30\% with VST (figure 2A), 50\% with CB106 (figure 2A) and $35 \%$ with GRP78 siRNA (figure 2B). The cell viability was only slightly decreased by the two down-regulators VST and CB106 (not shown). Fusion of cells was then measured in presence or not of down regulators of GRP78 or antibodies against GRP78 (AbC20).Treatment with antibodies against syncytin (AbSyn) known to inhibit fusion between trophoblast-derived cells [22] was used as positive control of decreased fusion capacity of BeWo cells. As shown in figure 3, down regulation of GRP78 by VST (figure 3A), CB106 (figure 3A) or GRP78 siRNA (figure 3B) significantly decreases forskolin-induced fusion of BeWo cells compared to control cells. Antibodies against syncytin and GRP78 (figure 3A) blocking syncytin and GRP78 membrane proteins respectively also decrease the fusion index of forskolin-treated BeWo cells. It would be noted that reverse transfection experiments has a negative impact on cell fusion which explains the difference of control values without forskolin between figure $3 \mathrm{~A}$ and $3 \mathrm{~B}$.

Since exofacial phosphatidylserine was described as a necessary component for cell fusion [23] [24], we have also evaluated phosphatidylserine efflux in BeWo cells. Similar to the effect on the fusion index, chemical down-regulators of GRP78 (figure 4A) or GRP78 siRNA (figure 4B) significantly decrease the efflux of phosphatidylserine induced by forskolin compared to control cells. Antibodies against syncytin and GRP78 seem to slightly decrease forskolin-induced efflux of phosphatidylserine compared to control cells (figure 4A). However, these results are not significant due to inter-experiments variability and proliferative effects of these antibodies.

\section{GRP78 down-regulation decreases forskolin-induced secretion of human chorionic gonadotrophin (hCG)}

Syncytialisation of cells is generally assessed by both cell fusion into syncytial unit and by induced secretion of hCG [5]. Indeed, differentiation of trophoblasts into syncytia is associated with increased production of hCG which can act in an autocrine manner to increase syncytium formation [25] [19]. We have thus measured hCG secretion in forskolin-induced BeWo cells transfected with GRP78 or control siRNA. As shown in figure 5, downregulation of GRP78 expression by siRNA in BeWo cells significantly decreases the forskolin-induced secretion.

\section{Expression of membrane GRP78 in preeclamptic CTBs compared to control CTBs}

PE has been already associated with defect of syncytialisation [26] [27]. We have thus evaluated expression and localization of GRP78 in control and PE CTBs. Table 1 reports the clinical information of women involved in this study. PE women had significantly elevated blood pressures and delivered earlier lower birthweight babies compared to normotensive patients (CTRL). Mean maternal age, parity and BMI were not significantly different between the 2 groups of patients.

Although GRP78 mRNA is significantly increased in PE CTBs compared to control cells (figure 6A), protein level of GRP78 measured by Cell-ELISA and confirmed by western blot (not shown) is not significantly different between control and PE cells (figure 6B). Interestingly, the proportion of GRP78 present at cell surface is significantly reduced in PE CTBs compared to control cells (figure 6C).

\section{Discussion}

Our previous investigation showed high GRP78 expression in villous CTBs, and particularly in STB [15] [16]. Here, we have found that GRP78 mRNA and protein expression are increased in first trimester CTB compared to term CTB suggesting the importance of GRP78 during embryo implantation. We had previously suggested its involvement in trophoblastic invasion [15], but its role in STB was never investigated. GRP78 expression is regulated by various stimuli such as hypoxia or glucose starvation [12] and can also be stimulated by cAMP [28]. cAMP has long been known to be a promoter of trophoblastic cell fusion by regulating several proteins such as syncytin 1 and 2. In the present study, treatment of BeWo cells with forskolin, used as inducer of cAMP, led to upregulation of GRP78 expression, concomitant with an increase in cell fusion. Moreover, down regulation of GRP78 led to a defective BeWo cell fusion and biochemical 
A-

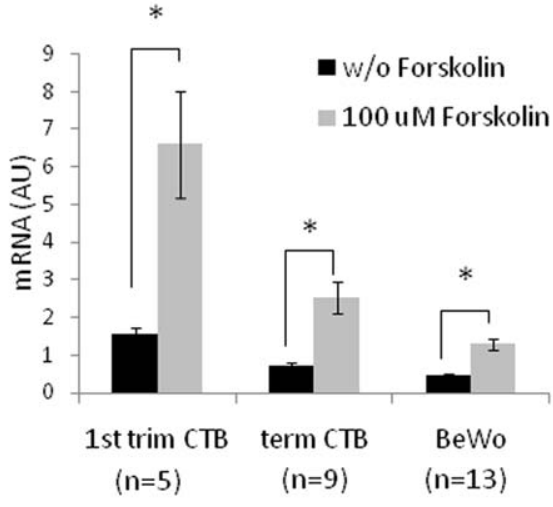

B-

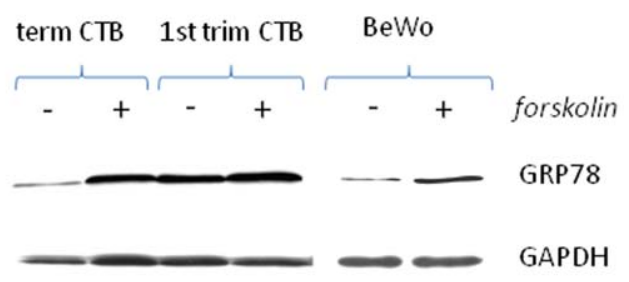

C-

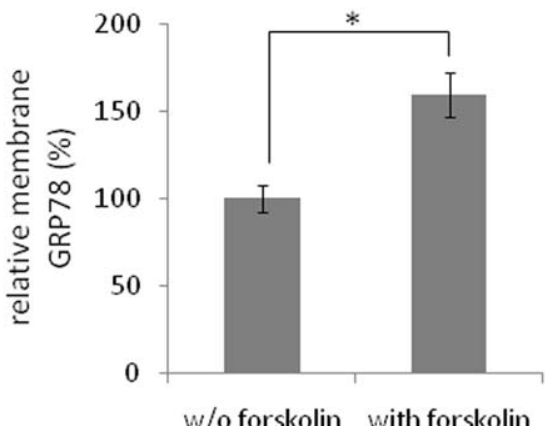

Figure 1. Effect of forskolin treatment on GRP78 expression in trophoblastic cells. Primary first trimester or term CTBs and BeWo cells were treated or not with $100 \mu \mathrm{M}$ forskolin for $96 \mathrm{~h}$ or $48 \mathrm{~h}$ respectively. A- Total RNA was then extracted, reverse transcribe andGRP78 CDNA was quantified by qPCR and normalized to cyclophilin A. B- Proteins ( $40 \mathrm{ug}$ ) were analysed by western blot with anti-GRP78 and anti-GAPDH antibodies. C- Membrane GRP78 expression was quantified by cell-ELISA in BeWo cells. Results are presented as mean \pm SEM. $n=3 * p<0.05$. doi:10.1371/journal.pone.0040596.g001

A-

$\mathrm{kDa}$
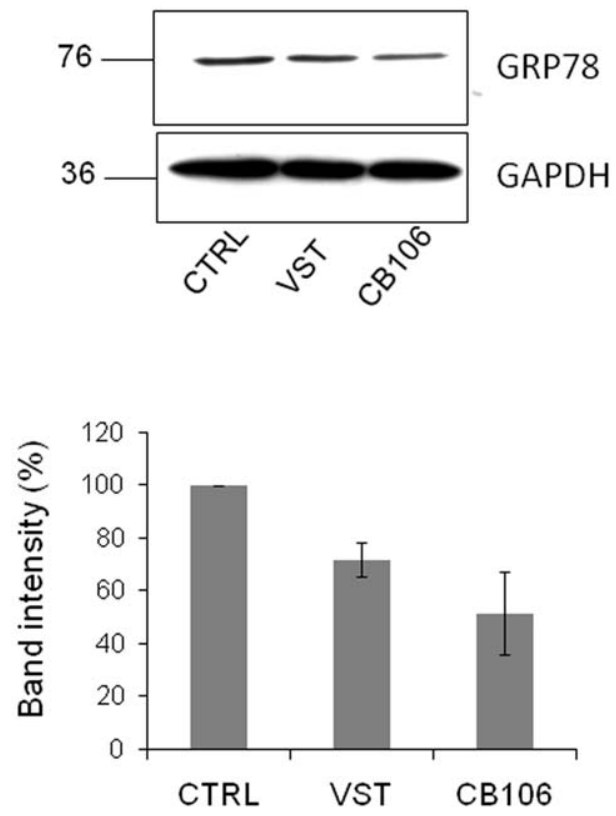

B-
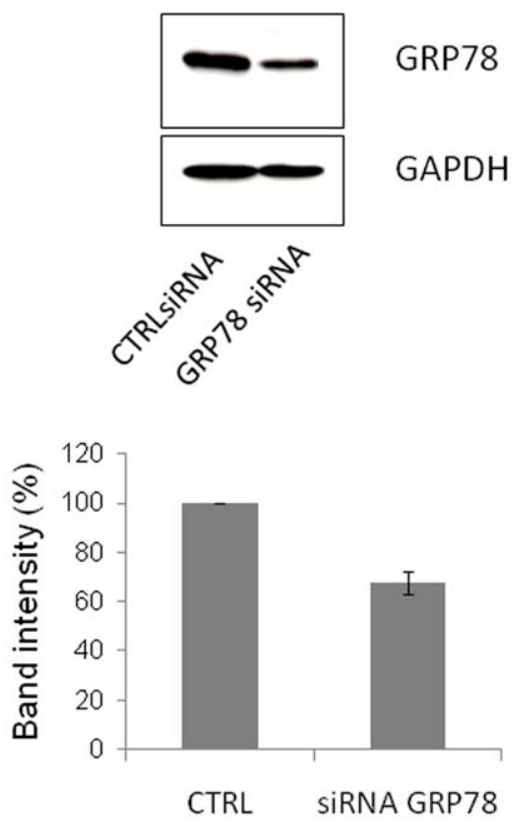

Figure 2. Downregulation of GRP78 in Bewo cells. A- Cells were untreated (CTRL) or treated with $20 \mu \mathrm{M}$ VST or CB106 for 48 h. B- Cells were transfected with GRP78 or control siRNA for $48 \mathrm{~h}$. A-B Upper panel: Immunoblots of GRP78 and GAPDH. Lower panel: The intensity of the GRP78 bands from three independent experiments was quantified and normalized to GAPDH. doi:10.1371/journal.pone.0040596.g002 
AW/o Forskolin 100 uM Forskolin

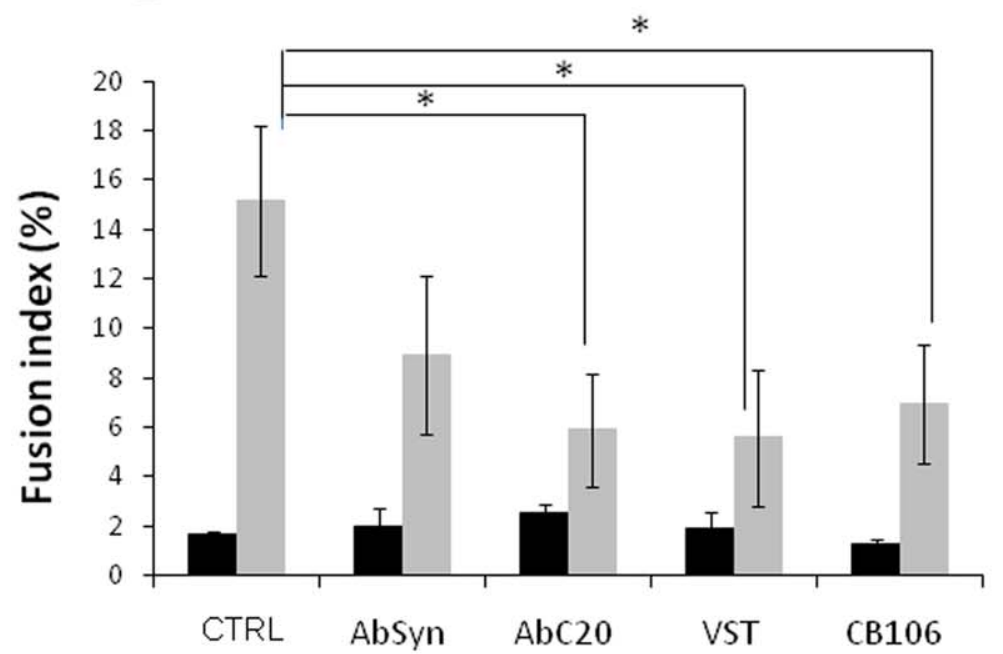

Bw/o Forskolin 100 uM Forskolin

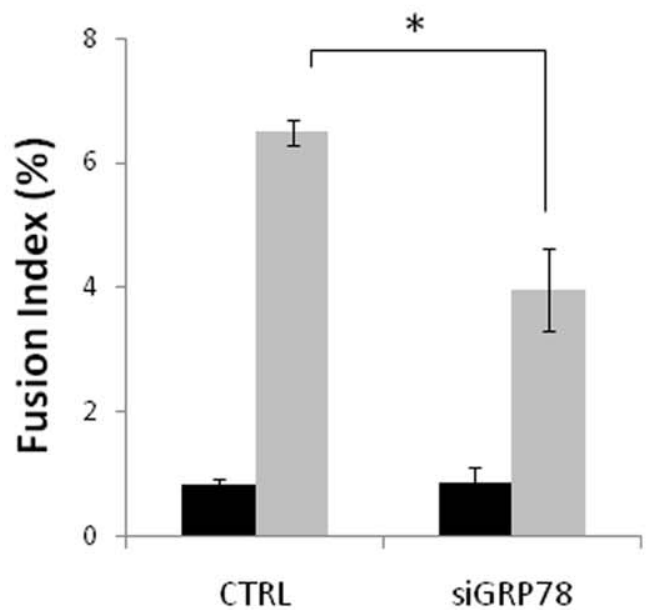

Figure 3. Role of GRP78 on fusion properties of BeWo cells. A- Cells were untreated (CTRL) or treated with anti-syncytin (AbSyn) or anti-GRP78 (AbC20) antibodies, $20 \mu \mathrm{M}$ VST or CB106 for $48 \mathrm{~h}$ in the absence or presence of $100 \mu \mathrm{M}$ forskolin. B- Cells were transfected with GRP78 or control siRNA for $48 \mathrm{~h}$ in presence or not of $100 \mu \mathrm{M}$ forskolin. Fusion index was calculated for 3 independent experiments. ${ }^{*} \mathrm{p}<0.05$. doi:10.1371/journal.pone.0040596.g003

differentiation, providing evidence that this protein is involved in syncytialisation of BeWo cells. As far as we know, among the long list of functions that have been attributed to GRP78, cell fusion had never been mentioned. Furthermore, the decreased capacity of BeWo cell fusion in the presence of anti-GRP78 antibodies strongly suggests that cell-surface GRP78 is a necessary component of trophoblastic cell differentiation. However, we have not yet identified a partner of GRP78 involved in this process and the mechanism of cell fusion linked to GRP78 remains to be investigated. Nevertheless, alpha 2 macroglobulin seems to be an interesting candidate if we consider signaling pathways involved in trophoblastic fusion $[19,29,30]$ and signaling pathways activated by alpha 2 macroglobulin binding to membrane GRP78 described in cancer cells [14].

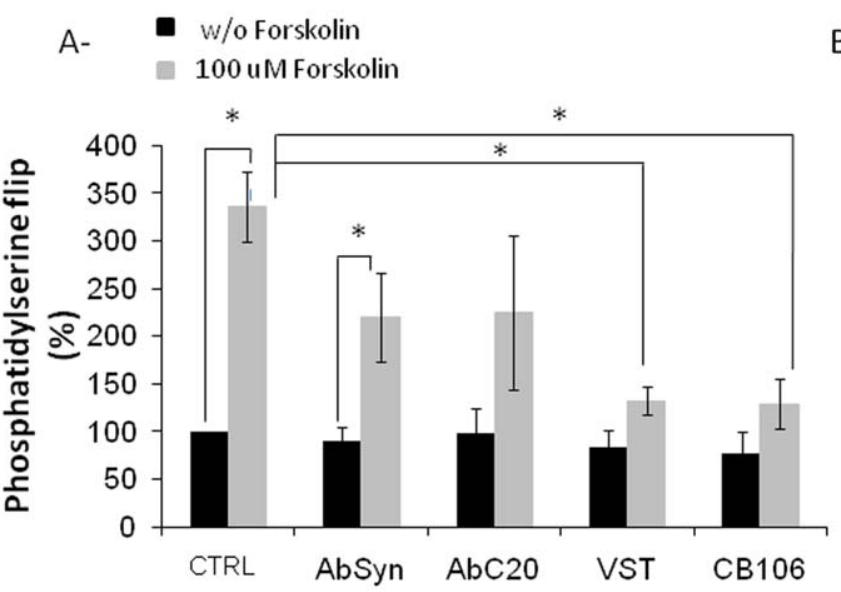

Several studies have highlighted abnormal trophoblastic differentiation in choriocarcinoma, PE, and a few other pathological conditions of pregnancy (for review, [8]). In PE, incomplete differentiation of CTBs to both invasive and villous CTBs was observed. As a consequence, there is shallow invasion of the pregnant uterus and defective syncytin expression in STB responsible for the characterized disturbances in STB in PE such as increased numbers of syncytial knots [31] [26].

In the present study, we have observed an apparent overexpression of GRP78 mRNA in PE GTBs which is consistent with the fact that GRP78 expression is induced by glucose deprivation and hypoxia, two characteristic features of PE. However GRP78 protein level is not modified in PE CTBs compared to control CTBs. This could be due to an increased turnover of GRP78 protein in PE CTBs compared to control CTBs. In contrast,
$B-$

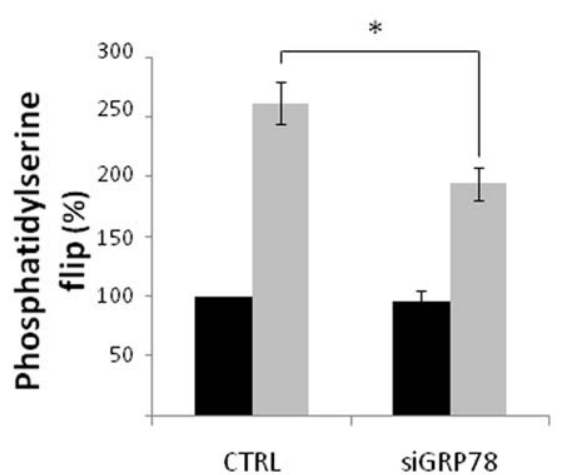

Figure 4. Role of GRP78 on phosphatidylserine flip of BeWo cells. A- BeWo cells were seeded on gelatin layer and treated or not with antisyncytin (AbSyn) or anti-GRP78 (AbC20) antibodies, $20 \mu \mathrm{M}$ VST or CB106 in the absence or presence of $100 \mu \mathrm{M}$ forskolin for $48 \mathrm{~h}$. B- BeWo cells were transfected with GRP78 or control siRNA and seeded on gelatin layer. The following day, cells were treated or not with forskolin for $48 \mathrm{~h}$. Phosphatidylserine flip was evaluated by a colorimetric assay (APOPercentage). $n=3,{ }^{*} p<0.05$. doi:10.1371/journal.pone.0040596.g004 


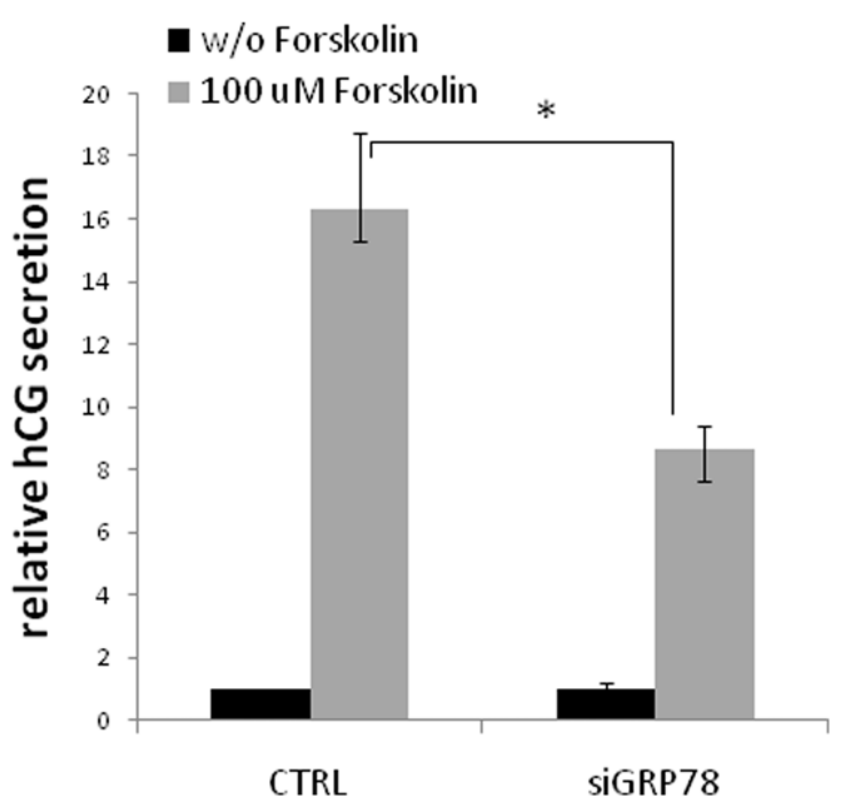

Figure 5. Effect of GRP78 down regulation on hCG secretion. BeWo cells were transfected with GRP78 or control siRNA and seeded on gelatin layer and then treated or not with $100 \mu \mathrm{M}$ forskolin for $48 \mathrm{~h}$. hCG level in culture supernatant was determined by ELISA and normalized to protein extract concentration. $n=3,{ }^{*} p<0.05$. doi:10.1371/journal.pone.0040596.g005

membrane GRP78 level is decreased in PE CTBs compared to control ones. This last observation could explain the reduced level of circulating GRP78 autoantibodies found in pregnant women who subsequently developed PE [16]. Moreover, since GRP78 protein level is not different between PE CTBs and control CTBs, the decreased membrane GRP78 expression observed in PE CTBs is probably due to an altered GRP78 translocation from the ER to the plasma membrane. At present, the mechanism allowing GRP78 to translocate to the different cellular compartments are not well understood. Indeed, overexpression of GRP78 can lead to cell surface localisation by saturation of KDEL retention mechanism, but ER stress can also actively promote GRP78 surface expression [32]. Moreover, relocalization of GRP78 to cell surface involves cell specific transporting proteins such as MTJ-I in macrophages [33] or Par-4 in prostate cancer PC-3 cells [34,35]. Therefore, the decreased expression of cell surface GRP78 in PE CTBs could be due to an altered expression of GRP78 trophoblastic transporting proteins. Mechanisms leading to impaired relocalisation of GRP78 at cell surface in PE cells have to be investigated to better understand the dysfunctions responsible for PE.

In conclusion, membrane GRP78 seems to be involved in trophoblastic cell fusion and biochemical differentiation in vitro. Moreover, we have highlighted a possible impaired mechanism of GRP78 relocation in PE CTBs which could be associated to defective syncytialisation in vivo. Together, these results strongly suggest the importance of membrane GRP78 in syncytialisation process.

\section{Materials and Methods}

\section{Ethics statement}

This research has been approved by the departmental ethics committee of maternity and pediatrics, University Hospital of
Table 1. Demographic and clinical characteristics of the study groups.

\begin{tabular}{llll}
\hline & & & \\
\hline & CTRL (n=9) & Severe PE (n=6) & p value \\
\hline Maternal age (year) & $33.2+/-1.8$ & $34.6+/-1.9$ & 0.66 \\
\hline Gestational age at delivery (week) & $37.2+/-0.9$ & $29.4+/-1$ & 0.0003 \\
BMI & $26.8+/-1.8$ & $27.4+/-3.1$ & 0.86 \\
Max systolic blood & $122.8+/-3.2$ & $166.8+/-7.6$ & 0.0003 \\
Max diastolic blood & $80.1+/-2.2$ & $106+/-4.8$ & 0.0006 \\
Parity & $0.9+/-0.3$ & 0 & 0.08 \\
Gravidity & $2.5+/-0.5$ & 1 & 0.04 \\
Baby weight (gram) & $3021+/-299$ & $1168+/-227$ & 0.002 \\
\hline doi:10.1371/journal.pone.0040596.t001 & &
\end{tabular}

Geneva (10-001 and 02-088). Informed written consent was obtained from all patients before their inclusion in the study.

\section{BeWo Cell culture}

BeWo cells (ATCG, CGL-98) were kindly provided by Dr Thierry Fournier (Inserm U767, Paris, France) and cultured at $37^{\circ} \mathrm{C}$ and $5 \% \mathrm{CO}_{2}$ in Ham's $\mathrm{F} 12 \mathrm{~K}$ medium (Gibco, Invitrogen, Basel, Switzerland) supplemented with 10\% FBS (Biochrom AG, Oxoid AG, Basel, Switzerland) and $0.05 \mathrm{mg} / \mathrm{ml}$ gentamycin (Invitrogen, Basel, Switzerland). Syncytialisation was induced $24 \mathrm{~h}$ after cells seeding of with $100 \mu \mathrm{M}$ forskolin (Sigma-, St Louis, MO, USA) for $48 \mathrm{~h}$. Control cells were treated with $1 \%$ ethanol (the vehicle for forskolin).

\section{Study groups}

Severe PE was diagnosed using standard definitions from hypertension defined as a systolic blood pressure level $\geq 160 \mathrm{mmHg}$ or a diastolic blood pressure level $\geq 110 \mathrm{mmHg}$ on two occasions and proteinuria $\geq 3+$ on a urine stick or $\geq 5 \mathrm{~g}$ in a 24-hour urine specimen (ACOG practice, 2002).

Nine control and 6 PE patients were recruited for this study. Their characteristics are given in Table 1.

\section{Purification of CTBs}

CTBs were isolated from first trimester trophoblast obtained after legal abortion and placentas obtained after delivery as described previously [36]. In brief, fresh tissue specimen were isolated and washed several times in sterile HBSS. Tissue was then enzymatically digested 5 times for $20 \mathrm{~min}$ at $37^{\circ} \mathrm{C}(0.25 \%$ trypsin, $0.25 \mathrm{mg} / \mathrm{ml}$ Dnase I). After incubation, the trypsin cocktail was neutralized with FBS, and the cells resuspended in DMEM (Gibco, Invitrogen, Basel; Switzerland). This cell suspension was filtered (100 $\mu \mathrm{m}$ mesh), laid onto a Percoll gradient $(70 \%$ to $5 \%$ Percoll diluted with HBSS) and centrifuged for $25 \mathrm{~min}$ at $1200 \mathrm{~g}$. The $30-45 \%$ percoll layer containing CTBs was collected, the cells washed and resuspended in DMEM. Cells were then immunopurified with immobilized anti-CD45 antibodies

\section{Real-time quantitative PCR}

RNA was extracted using RNeasy mini kit (QIAGEN, Basel, Switzerland). Reverse transcription was performed with $400 \mathrm{ng}$ of total RNA using QuantiTect Reverse Transcription kit (QIAGEN). The quantitative detection of the PCR product was performed using the qPCR Mastermix Plus for SYBR Green I (Eurogentec, Seraing, Belgium), supplemented with fluorescein (Bio-Rad, Reinach, Switzerland), with the iCycler iQ System (Bio- 
A-

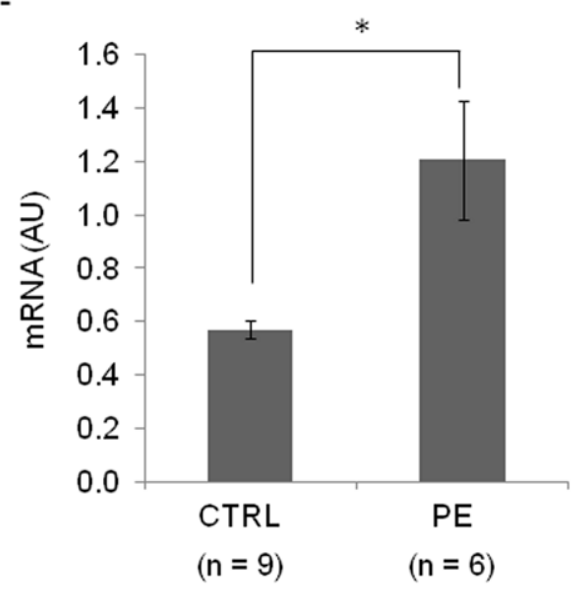

B-

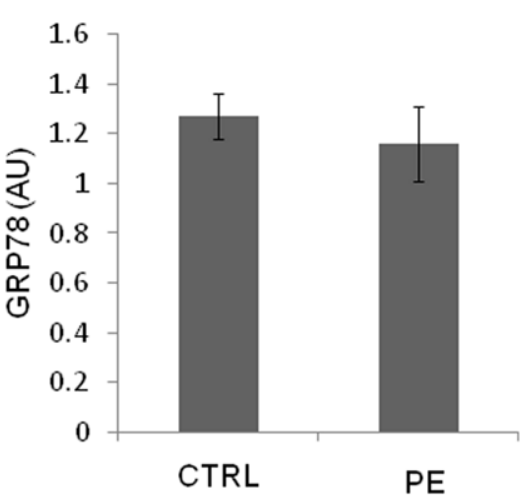

C-

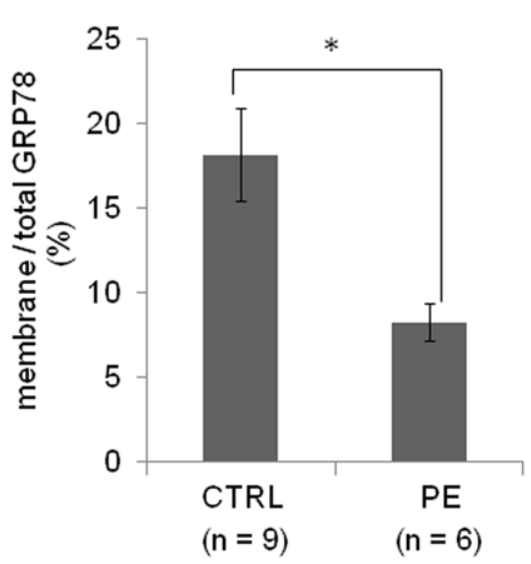

Figure 6. GRP78 expression in trophoblastic cells. A- GRP78 mRNA from control (CTRL) and preeclamptic (PE) trophoblastic cells was quantified by qPCR and normalized to cyclophlin A. B- GRP78 expression from control (CTRL) and preeclamptic (PE) trophoblastic cells was quantified by Cell-ELISA. C- Relative proportion of membrane GRP78 in trophoblastic cells purified from control (CTRL) and preeclamptic (PE) trophoblastic cells was evaluated by Cell-ELISA. Results are presented as mean \pm SEM. $n=3 * p<0.05 ; A U$ : arbitrary unit. doi:10.1371/journal.pone.0040596.g006

Rad). The relative expression of GRP78 was normalized to the housekeeping gene cyclophilin A. Oligonucleotide primers for qPCR were as follows: human cyclophilin A forward $5^{\prime}$ TACGGGTCGTGGCATCTTGT-3' and reverse $5^{\prime}-$ CAATTTGTGTTGGGTCCAGC-3', human GRP78 forward 5'-CGTGGAGATCATCGCGAAC-3' and reverse 5'-ACATAGGACGGGGTGATGG-3'.

\section{siRNA transfection}

Cells were plated at $3 \times 10^{5}$ cells into 6 -well plates or at $3 \times 10^{4}$ cells into 96-well plates and immediately transfected with $40 \mathrm{nM}$ of GRP78 siRNA (Santa Cruz Biotechnology, Labforce, Nunningen, Switzerland) or control siRNA (SiRNA-A, Santa Cruz Biotechnology). Transfection of siRNA was carried out using Lipofectamine 2000 (Invitrogen, Basel, Switzerland) following the reverse transfection protocol provided by the manufacturer.

\section{Western Blot}

Whole BeWo cell extracts (40 $\mu \mathrm{g}$ of proteins) were fractionated by SDS-Page $10 \%$ and transferred to nitrocellulose membrane for immunoblot analysis using rabbit anti-GRP78 antibodies (GL-19, 1:3000 dilution from Sigma) and mouse anti-GAPDH antibodies (1:30 000 dilution from Millipore, Temecula, CA, USA).

\section{Fusion index}

To visualize syncytia, BeWo cells were fixed in methanol at $-20^{\circ} \mathrm{C}$ for $30 \mathrm{~min}$ and incubated in PBS containing 3\% BSA for
$30 \mathrm{~min}$ to eliminate non-specific binding. Cells were then incubated in the presence of goat anti-desmoplakin antibodies (1:50 dilution, from Santa Cruz Biotechnology,) overnight at $4^{\circ} \mathrm{C}$, washed with PBS and incubated with anti-goat IgG-HRP (1:500 dilution, from Santa Cruz Biotechnology) for $1 \mathrm{~h}$ at room temperature. After washes in PBS, staining was revealed in diaminobenzidine (Dako).

The fusion index expressed in percent was calculated as follows: $[(\mathrm{N}-\mathrm{S}) / \mathrm{T}] \times 100$, where $\mathrm{N}$ equals the number of nuclei in syncytia, $\mathrm{S}$ equals the number of syncytia and $\mathrm{T}$ equals the total number of nuclei counted. This index was calculated for 3 independent experiments, run in triplicate.

\section{Phosphatidylserine flip}

Bewo cells were seeded on gelatine layer and treated with forskolin as mentioned before. Phosphatidylserine flip was evaluated in 3 independent experiments, run in triplicate by a colorimetric assay (APOPercentage from Biocolor Life Science, UK) following instructions of manufacturer.

\section{Cell-ELISA of membrane GRP78}

Cell-ELISA was performed on CTBs as previously described [16]. Relative proportion of membrane GRP78 was calculated by dividing absorbance of membrane GRP78 (measured on nonpermeabilized cells) over total GRP78 (measured on permeabilized cells) for 3 independent experiments run in triplicate. 


\section{ELISA of hCG}

BeWo cells were transfected with control or GRP78 siRNA and then cultured for $48 \mathrm{~h}$ in the presence or not of $100 \mu \mathrm{M}$ of forskolin before culture medium was collected and centrifuged for $5 \mathrm{~min}$ at $14000 \mathrm{~g}$. Levels of hCG in supernatants were determined by enzyme immunoassay (ELISA; DRG International, Diagnostik Medizintechnik, Oberdof, Switzerland) following manufacturer's instructions and normalized by total cellular protein content of corresponding wells.

\section{Statistical analysis}

Data were expressed as means \pm SEM for $\mathrm{n}$ different samples. Statistical differences between samples were assessed by the Student's t test and the $\mathrm{p}$ value $<0.05$ was considered significant.

\section{References}

1. Yoshie M, Kaneyama K, Kusama K, Higuma C, Nishi H, et al. (2010) Possible role of the exchange protein directly activated by cyclic AMP (Epac) in the cyclic AMP-dependent functional differentiation and syncytialization of human placental BeWo cells. Hum Reprod 25:2229-38.

2. Al-Nasiry S, Spitz B, Hanssens M, Luyten G, Pijnenborg R (2006) Differential effects of inducers of syncytialization and apoptosis on BeWo and JEG-3 choriocarcinoma cells. Hum Reprod 21:193-201.

3. Pollheimer J, Knofler M (2005) Signalling pathways regulating the invasive differentiation of human trophoblasts: a review. Placenta 26 Suppl A:S21-30.

4. Ferretti C, Bruni L, Dangles-Marie V, Pecking AP, Bellet D (2007) Molecular circuits shared by placental and cancer cells, and their implications in the proliferative, invasive and migratory capacities of trophoblasts. Hum Reprod Update 13:121-41.

5. Potgens AJ, Drewlo S, Kokozidou M, Kaufmann P (2004) Syncytin: the major regulator of trophoblast fusion? Recent developments and hypotheses on its action. Hum Reprod Update 10:487-96.

6. Bischof P, Irminger-Finger I (2005) The human cytotrophoblastic cell, a mononuclear chameleon. Int J Biochem Cell Biol 37:1-16.

7. Keryer G, Yassenko M, Labbe JC, Castro A, Lohmann SM, et al. (1998) Mitosis-specific phosphorylation and subcellular redistribution of the RIIalpha regulatory subunit of cAMP-dependent protein kinase. J Biol Chem 273:34594 602.

8. Handwerger S (2010) New insights into the regulation of human cytotrophoblast cell differentiation. Mol Cell Endocrinol 323:94-104.

9. Dudek J, Benedix J, Cappel S, Greiner M, Jalal C, et al. (2009) Functions and pathologies of BiP and its interaction partners. Cell Mol Life Sci 66:1556-69.

10. Gonzalez-Gronow M, Selim MA, Papalas J, Pizzo SV (2009) GRP78: a multifunctional receptor on the cell surface. Antioxid Redox Signal 11:2299306.

11. Fu Y, Lee AS (2006) Glucose regulated proteins in cancer progression, drug resistance and immunotherapy. Cancer Biol Ther 5:741-4.

12. Li J, Lee AS (2006) Stress induction of GRP78/BiP and its role in cancer. Curr Mol Med 6:45-54

13. Pfaffenbach KT, Lee AS (2011) The critical role of GRP78 in physiologic and pathologic stress. Curr Opin Cell Biol 23:150-6.

14. Ni M, Zhang Y, Lee AS (2011) Beyond the endoplasmic reticulum: atypical GRP78 in cell viability, signalling and therapeutic targeting. Biochem $\mathrm{J}$ 434:181-8.

15. Arnaudeau S, Arboit P, Bischof P, Shin-ya K, Tomida A, et al. (2009) Glucoseregulated protein 78: a new partner of p53 in trophoblast. Proteomics 9:531627.

16. Laverriere A, Landau R, Charvet I, Irion O, Bischof P, et al. (2009) GRP78 as a marker of pre-eclampsia: an exploratory study. Mol Hum Reprod 15:569-74.

17. Kudo Y, Boyd CA, Sargent IL, Redman CW (2003) Hypoxia alters expression and function of syncytin and its receptor during trophoblast cell fusion of human placental BeWo cells: implications for impaired trophoblast syncytialisation in pre-eclampsia. Biochim Biophys Acta 1638:63-71.

18. Kudo Y, Boyd CA, Kimura H, Cook PR, Redman CW, et al. (2003) Quantifying the syncytialisation of human placental trophoblast BeWo cells grown in vitro. Biochim Biophys Acta 1640:25-31.

\section{Acknowledgments}

We wish to thank Antonina Chilin for enrolling patients and collecting placentas and Paul Bischof for his helpful discussion.

\section{Author Contributions}

Conceived and designed the experiments: SF SP PR MC. Performed the experiments: SF SP PR MC. Analyzed the data: SF SP PR MC. Contributed reagents/materials/analysis tools: SF MC. Wrote the paper: SF SP PR MC ME KS OI.

19. Delidaki M, Gu M, Hein A, Vatish M, Grammatopoulos DK (2011) Interplay of cAMP and MAPK pathways in hCG secretion and fusogenic gene expression in a trophoblast cell line. Mol Cell Endocrinol 332:213-20.

20. Wice B, Menton D, Geuze H, Schwartz AL (1990) Modulators of cyclic AMP metabolism induce syncytiotrophoblast formation in vitro. Exp Cell Res 186:306-16

21. Park HR, Tomida A, Sato S, Tsukumo Y, Yun J, et al. (2004) Effect on tumor cells of blocking survival response to glucose deprivation. J Natl Cancer Inst 96:1300-10.

22. Mi S, Lee X, Li X, Veldman GM, Finnerty H, et al. (2000) Syncytin is a captive retroviral envelope protein involved in human placental morphogenesis. Nature 403:785-9.

23. Adler RR, Ng AK, Rote NS (1995) Monoclonal antiphosphatidylserine antibody inhibits intercellular fusion of the choriocarcinoma line, JAR. Biol Reprod 53:905-10.

24. Das M, Xu B, Lin L, Chakrabarti S, Shivaswamy V, et al. (2004) Phosphatidylserine efflux and intercellular fusion in a BeWo model of human villous cytotrophoblast. Placenta 25:396-407.

25. Shi OJ, Lei ZM, Rao CV, Lin J (1993) Novel role of human chorionic gonadotropin in differentiation of human cytotrophoblasts. Endocrinology 132:1387-95.

26. Vargas A, Toufaily C, Lebellego F, Rassart E, Lafond J, et al. (2011) Reduced Expression of Both Syncytin 1 and Syncytin 2 Correlates With Severity of Preeclampsia. Reprod Sci.

27. Langbein M, Strick R, Strissel PL, Vogt N, Parsch H, et al. (2008) Impaired cytotrophoblast cell-cell fusion is associated with reduced Syncytin and increased apoptosis in patients with placental dysfunction. Mol Reprod Dev 75:175-83.

28. Alexandre S, Nakaki T, Vanhamme L, Lee AS (1991) A binding site for the cyclic adenosine $3^{\prime}, 5^{\prime}$-monophosphate-response element-binding protein as a regulatory element in the grp78 promoter. Mol Endocrinol 5:1862-72.

29. Daoud G, Amyot M, Rassart E, Masse A, Simoneau L, et al. (2005) ERK1/2 and p38 regulate trophoblasts differentiation in human term placenta. J Physiol 566: 409-23.

30. Vaillancourt C, Lanoix D, Le Bellego F, Daoud G, Lafond J (2009) Involvement of MAPK signalling in human villous trophoblast differentiation. Mini Rev Med Chem 9:962-73.

31. Matthiesen L, Berg G, Ernerudh J, Ekerfelt G, Jonsson Y, et al. (2005) Immunology of preeclampsia. Chem Immunol Allergy 89:49-61.

32. Zhang Y, Liu R, Ni M, Gill P, Lee AS (2010) Cell surface relocalization of the endoplasmic reticulum chaperone and unfolded protein response regulator GRP78/BiP. J Biol Chem 285:15065-75.

33. Misra UK, Gonzalez-Gronow M, Gawdi G, Pizzo SV (2005) The role of MTJ-1 in cell surface translocation of GRP78, a receptor for alpha 2-macroglobulindependent signaling. J Immunol 174:2092-7.

34. Burikhanov R, Zhao Y, Goswami A, Qiu S, Schwarze SR, et al. (2009) The tumor suppressor Par-4 activates an extrinsic pathway for apoptosis. Cell 138:377-88.

35. Lee AS (2009) The Par-4-GRP78 TRAIL, more twists and turns. Cancer Biol Ther 8:2103-5.

36. Bischof P, Martelli M, Campana A, Itoh Y, Ogata Y, et al. (1995) Importance of matrix metalloproteinases in human trophoblast invasion. Early Pregnancy $1: 263-9$. 